\title{
Expansiones de la clasificación del Congreso para México, clase F
}

\author{
Roberto Abell Bennett \\ Investigador del CUIB
}

Han aparecido varias expansiones valiosas para la ampliación de la clase F, referente a la literatura bibliográfica sobre México en las adiciones y cambios de la Biblioteca del Congreso (1928 - ), acumulados por el Gale Research Compañy (1974- ). Algunos cambios son específicamente para nuestro país y otras adiciones se puede aplicar a la historia y geografía local mexicana en su caso. En le ejemplo de México y los países de América Latina, la clase F (Library of Congress 1958) desarrolla únicamente bloques de números, además no existía todavía a principios de siglo la práctica de desarrollar dobles letras para los países individuales como hicieron después para la clase D, historia general y del Viejo Mundo (Library of Congress 1959). Por lo tanto existen apenas doscientos números para México. La adición de una segunda letra a la clasificación la hubiera aumentado a diez mil números mexicanos. Por ejemplo, para cada país importante europeo se tiene desglosado unos mil números y esto ha permitido una ampliación para nuevos países creados después de la Primera Guerra Mundial. La literatura histórica y geográfica mexicana ha crecido bastante principios de siglo. Lo que ha restringido las necesidades de las bibliotecas mexicanas.

En el esquema de la clase F, se han dado expansiones locales para México. No se les ha tomado en cuenta para este trabajo por desviarse demasiado de la Clasificación del Congreso, se ha considerado primordial seguirla para no bloquear expansiones futuras de la Biblioteca del Congreso aceptado en un plano internacional. 
Debido a la abundancia de obras sobre la geografía mexicana en última fechas, la Biblioteca del Congreso ha cerrado el periodo 1951 - 1980 asignado a F1216 y ha creado un nuevo número, F1216.5 para materiales bibliográficos tratados desde 1981 en adelante (cuadro 1). Como ocurre en la Clasificación del Congreso, existen números sin usar y por lo tanto fue fácil abrir este número, aún siendo decimal, para obras de geográficas actuales. Ha habido necesidad en estos esquemas sobre América Latina de crear muchos números decimales debido a la estrechez del esquema y la falta de número enteros en su caso que pudieran ser asignados a algún tema nuevo. Este problema se crea una vez más al no haber asignado una segunda letra sino bloques reducidos de números a cada país ( Immroth 1980, p.168).

Referente a la arqueología mexicana ha ocurrido un intento especial para poner al día los esquemas (cuadro 2) . ha habido una serie de cambios importantes para desalojar materiales sobre códices, arqueología prehispánica y culturas precolombina específicas con nuevas subdivisiones, hasta entonces clasificados en un solo número, F1219.

Para congresos, museos y exposiciones, no solo se ha agregado F1202 para congresos, ampliado F1203 para incluir museos y exhibiciones mexicanas generales (cuadro 1) sino, se ha creado el número f1218.5 para museos y exposiciones arqueológicas (cuadro 2). También van incluidos en este número exposiciones sobre los indígenas mexicanos contemporáneo.

Para los sitios arqueológicos específicos, F1219.A-Z, se da preferencia a la clasificación por estado en vez de un simple listado alfabético de sitios locales (cuadro2). Es decir, la Biblioteca del congreso favorece la localización de sitios por áreas culturales. Aunque los estados actuales no engloban con tanta precisión estas áreas, por lo menos es mejor que un listado de sitios locales puramente alfabético. Por ejemplo, para Oaxaca la Clasificación del Congreso asigna F1219.011 a menos que ya tenga números de Cutter propios, por ejemplo para Mitla, .M6. Es dudoso que el estado actual de Oaxaca resulte ser una sola región cultural, pues incluye además de la zona Zapoteca a la Mixteca y en el estado de Puebla se podría incluir la Mixteca Baja en esta área 
cultural. Además, se traslapa en cierto modo con el listado de culturas antiguas enF1219.8. sin embargo, es un importante paso hacia delante.

Los tópicos específicos, F1219.3. A-Z son para varias etnias indígenas mexicana (cuadro 2). Mientras se clasifican obras sobre arte, implementos, vivienda, etcétera con el sitio determinados en F1219.1.A-Z, se reserva a los Azteca, F1219 o de otra cultura prehispánica F1219.8.A-Z y para tópicos especiales de los pueblos actuales, F1221. A-Z. Por ejemplo, se coloca una obra sobre la cerámica de Monte Albán en F1219.1.011; el arte Azteca en F1219.76.A78; la escultura Olmeca en F1219.8.056 y la indumentaria Chinanteca moderna en F1221.Ch56. Existen muchos números más para estos temas de los que anteriormente, había.

Es muy útil tener números específicos sobre los códices, ahora en F1219.5F1219.56. De cualquier manera, poco material entra en F1219.54 y tomando en cuenta que existen más de 150 códices, podría haber congestionamiento en F1219.56, número para los códices individuales.

Ahora la mayoría de las obras sobre la civilización prehispánicas se clasifican en F1219.7, por ejemplo, las obras de Clavijero, Motolinía y Torquemada por mencionar algunas (cuadro 2). Puede observarse que en F1219 caben notablemente menos obras que antes.

Libros sobre los Mixicas, conocidos tal vez mejor como Aztecas, se colocan en F1219.73-F1219.76 incluyendo números para biografía y tópicos especiales únicamente aplicados a ellos (cuadro2). Las otras culturas sobre las cuales se tendría menos material, se arreglan alfabéticamente según su número de Cutter para cada una en F1219.8.A-Z. Esto resulta mejor que asignar toda esta literatura a un solo número en F1219. Los grupos modernos se clasifican en F1220 si son obras generales y en F1221 sobre grupos específicos. Como ya se indicó, se clasificarían todos los temas específicos sobre estas culturas en el mismo lugar, es de suponer que los investigadores así lo prefieren.

Existen una desconcentración algo desordenada sobre las obras de los Mayas, en vez de clasificarlos con México, la Biblioteca del Congreso coloca las obras generales sobre este grupo, de sus sitios arqueológicos específicos y de sus 
tópicos especiales en F1435-F1435.3, números para los indígenas y arqueología centroamericana. Para obras generales pero relacionadas con los Mayas de la región de Belice, Guatemala y Yucatán existe no sólo el número F1376 sino también F1219.1.Y8. Para tener juntas todas las obras generales sobre los Mayas de Yucatan, sería mejor, supuestamente, escoger uno de los dos números.

Referente a la historia mexicana, la Biblioteca del Congreso (1928-List 217, January -March 1985) ha ampliado sustancialmente en las adiciones y cambios la Lista de las regiones y países en alfabeto único que se utiliza para las relaciones exteriores con países específicos en F1228.5.A-Z (cuadro 3), de la cual se tiene las traducciones necesarias (Abell 1987). Al contrario, para las obras generales sobre la historia diplomática se siguen clasificando en F1228 y para la literatura sobre las relaciones exteriores por periodo, se continua colocándolas con su etapa respectiva en F1219-F1236.

La Biblioteca del Congreso ha suplido con un número nuevo, F1228.98, para el período de los primeros contactos españoles desde el descubrimiento de América hasta 1519 (cuadro 3). Para épocas recientes, es decir, posteriormente a 1946, ya existen nuevos números para la biografía del período 1946-1970, F1235.5 y para la época actual no sólo para la biografía sino para obras generales en F1236-F1236.6. Esto ayuda mucho a descongestionar las obras sobre períodos actuales. No sólo esto, sino con los números biográficos se deben utilizar la tabla XI para los períodos recientes y para toda la historia mexicana por época, F1230-f1234 en donde existen instrucciones de utilizarla para biografía, además de F1225.A3-Z, biografía de historiadores.

Se tendría que tomar precauciones sobre el material biográfico en estas etapas subsiguientes. Para las obras sobre la Guerra de 1847, quizá las bibliotecas mexicanas prefieren clasificar este material con la historia mexicana y no con los Estados Unidos a menos que el tema sea plenamente norteamericano. Por ejemplo, se colocaría en F1232 una obra sobre las campañas en México de Winfield scott, pero su autobiografía se clasificaría con la historia norteamericana. Por ende, obras sobre el período de la Reforma hasta el fin de la intervención en F1233 por la ambigüedad de citar en el esquema el primero con las fechas 1849-1858/1861 y el segundo por las fechas 1849/1861-1867. Sin em- 
bargo, se debe tener cuidado al clasificar la biografía hasta la reformas en F1232.5 y de la intervención en F1233 con el uso de la tabla XI, dado que los números son más específicos para esta materia.

Para la historia y geografía local mexicana la clasificación del Congreso asigna determinados números nuevos o instrucciones que aclaran el uso de algunos temas (cuadro4). Por ejemplo, se instruye al clasificador sobre el empleo del término Baja California, no sólo para el estado de Baja California sino que también para la Baja California Sur en F1246. La cuenta del Río Balsas tiene creado el nuevo número, F1247. Para el Valle de México existe un número reciente, F1302. También las Islas Revillagigedo y de San Juan de Ulúa tienen asignado nuevos números, F1233.5 y F1335 respectivamente. Para la Sierra Madre se deben clasificar obras sobre esta cordillera en F1340. El número para el Valle de México es tal vez el más importante respecto a las nuevas expansiones por el volumen de material que se agruparía aquí.

En el caso de ciudades y pueblos mexicanos se utilizan hoy en día para dos casos las tablas auxiliares V y VI. A éstos se ha asignado un número entero que se usa con la tabla V y para aquelios que han sido asociado a un número Cutter, se aprovecha la tabla VI (cuadro 4). En este caso hay instrucciones al respecto de usar las tablas y si no existen, no se deben utilizar. Por ejemplo, en adicciones y cambios se encuentra un número romano después de México, D.F. entre paréntesis (Tabla V) y después del número de Cutter para Monterrey también se da la misma indicación (Tabla VI). Hasta la fecha únicamente con estas dos ciudades se deben usar las tablas señaladas. La tabla V está diseñada para obras de forma en primera instancia con números reservados de Cutter y la tabla VI con números de Cutter para los demás temas. En la tabla V existen además números decimales para las divisiones generales y luego para secciones y distritos, monumentos y estatuas, parques y glorietas, calles y para edificios, además se señala el uso conjunto de otra tabla incluida en una nota de pie de página. De igual forma se procede con el uso de la tabla VI. Por ejemplo, según la nota, un libro sobre las calles de Monterrey escrito por Covarrubias, tomaría el número F1391.M775A23, el primer dígito "2" del segundo número reservado de Cutter para calles tratados colectivamente y el "3" por el asiento principal. En general los número F1386 y F1391 amplían con las tablas V y VI 
algunos detalles que permiten una gran expansión potencial para los materiales bibliográficos de las ciudades con más literatura referente a México.

Se habló en la II Reunión Nacional sobre Normalización del uso del Sistema de Clasificación del Congreso de los Estados Unidos (1986) de propuestas de modificación a este esquema de Clasificación, que de ahora en adelante se van a discutir en grupos reducidos de análisis. Un representante institucional que deberá ser jefe de procesos técnicos de un grupo selecto de instituciones se invitará a estas reuniones.

Se ha entre otras que se utilice la tabla VII para determinados estados mexicanos, agrupando las ciudades y pueblos por estados bajo el rubro Local, A-Z, el mismo proceder en general que se hace con ciudades y pueblos norteamericanos y canadiense (cuadro 5). Además se aplicará la tabla VI cuando sea conveniente a ciudades determinadas dentro del estado, no restringida tanto como hace la Biblioteca del Congreso hoy en día.

Por ejemplo, una biblioteca yucateca quizá prefiera utilizar la tabla VII, sin limitar como lo hace la Clasificación del Congreso, el uso de esta tabla a algunas islas del Caribe, aprovechando el número F1376 para la historia y geografía de Yucatán (cuadro 5) se tendrían que tomar en cuenta otros números yucatecos ya asignados en el esquema como ocurre con la arqueología maya. Por ejemplo la literatura sobre la arqueología e indígena yucatecos se clasificaría en F1376.3 según la tabla, pero existe el número F1219.1.y8 para el mismo tema. Además se podría clasificar la historia y descripción de ciudades y pueblos yucatecos en F1376.9.A-Z según la tabla VII que conformaría la práctica de clasificar materiales de ciudades de Estados Unidos y Canadá por estado y provincia. No solo eso, sino que se podría aplicar la tabla VI a ciudades yucatecas en F1376.9. A-Z hasta donde fuera necesario por el volumen de literatura. Tal vez se debe tener cuidado de no utilizar estas tablas de forma discriminada, por la elevación de costos que esto implicaría al no aceptar la signatura topográfica encontrada en el NUC o en Librunam y por el peligro de bloquear la posible expansión en el futuro de los esquemas de la Biblioteca del congreso. 
Como no se ve un futuro previsible la expansión a dos letras de la Clasificación del Congreso para México y otros países latinoamericanos, estamos con el problema de utilizar del modo más eficaz los doscientos números que tenemos para México. Con la expansión en el esquema de la geografía y la arqueología mexicana, el uso de la tabla bibliográfica para la historia mexicana además de la asignación de números nuevos para la historia actual y los posibles reajustes de la historia y geografía locales, se tiene a la mano una expansión dentro de los cánones de la Clasificación del Congreso para materiales históricos y geográficos mexicanos.

\section{Obras citadas}

1. ABELLB., Roberto. Manual para el manejo y aplicación de la Clasificación del Congreso a la historia, clase C, D, y E-F. México, UNAM, Centro Universitario de Investigaciones Bibliotecológicas, 1986 (en prensa)

2. IMMROTH, John Phillip. Immroth's quide to the Library of Congress clasification. $3^{\text {xd }}$ ed. Edite by Lois Mai Chan. Littleton, Colo., Libraries Unlimited, 1980 . $-402 \mathrm{p}$.i

3. Library of Congress. Subject Cataloging Division. LC classification, andditions and change (1928-) list 1-

4. Library of Congress. Subject Cataloging Division. Classification, History, Class E-F, Ameríca $3^{\text {rd }}$ ed. (with supplemetary pages) Washington : Library of Congress 1958 (reprinted 1965). -607p.

5. Library of Congress. Subject Cataloging Division. Classification, History, Class D, General and Old World. $2^{\text {nd }}$ ed. (with supplemetary pages) Washington: Library of Congress 1959 (reprinted 1966) . - - 747 p.

6. Library of Congress. Subject Cataloging Division. Library of Congress clasification schedules, a cumulation of additions and changes. Detroit, Gale Research Col, 1974-

7. Reunión Nacional sobre Normalización del uso del Sistema de Clasificación del Congreso de los Estados Unidos. (3a, 1985, México, D.F.). Reunión de trabajo : 8 de noviembre de 1985. Coordinación y edición : María Luisa Garza Avalos. México, CUIB, 1986 (en prensa)

1 Referencias ordenadas por fecha 
CUAORO 1

\section{LA GEOGRAFIA MEXICAMIA}

\section{LAIIM AMERICA. SPAMISH AMERICA}

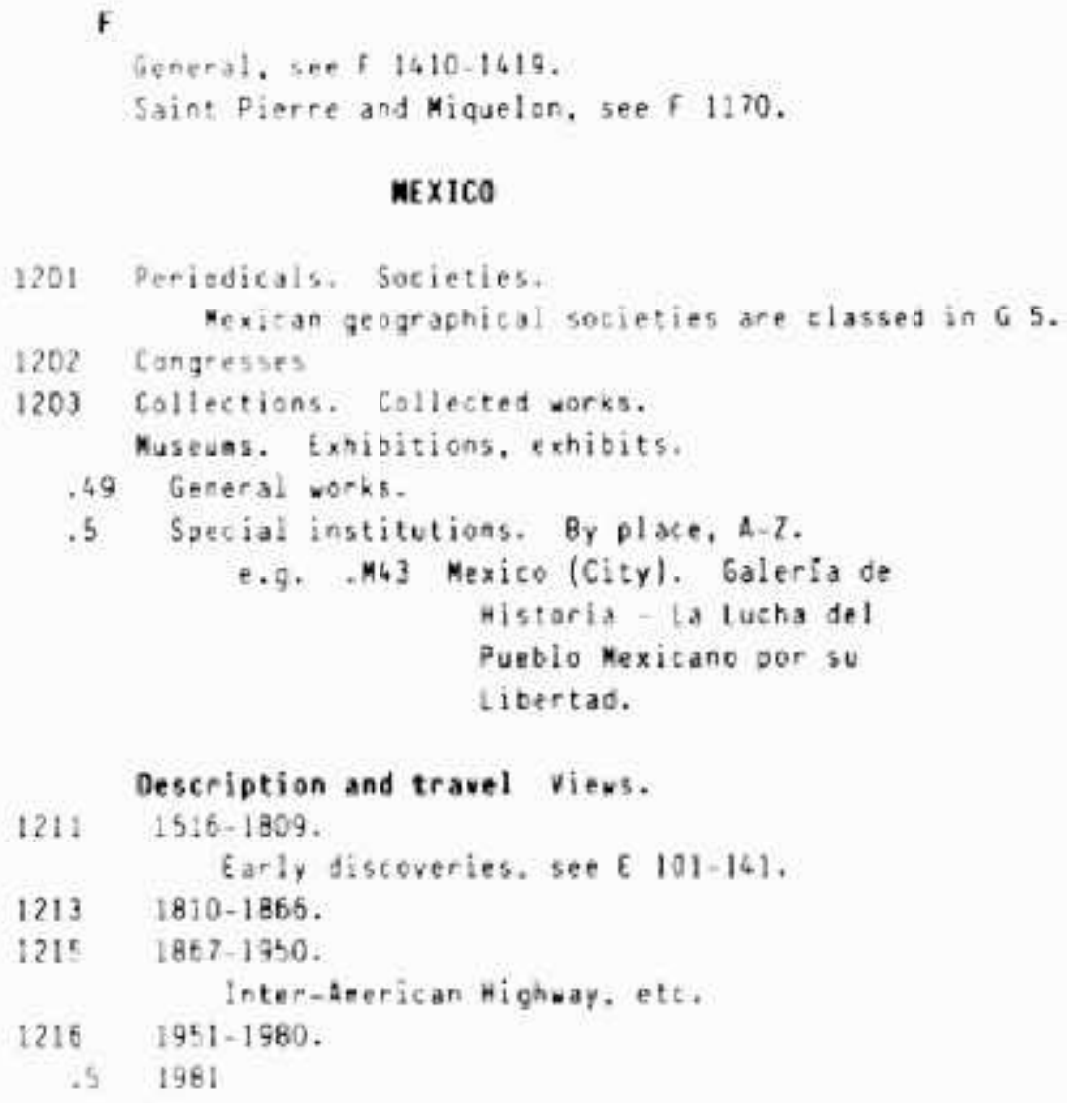




\section{CUADRO 2}

\section{AMIROPOLOGIA mEXICAMa}

\section{MEXICO}

1218.5

1719

$+1$

.3

.5

.54

.56

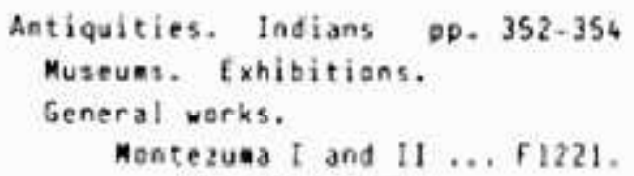




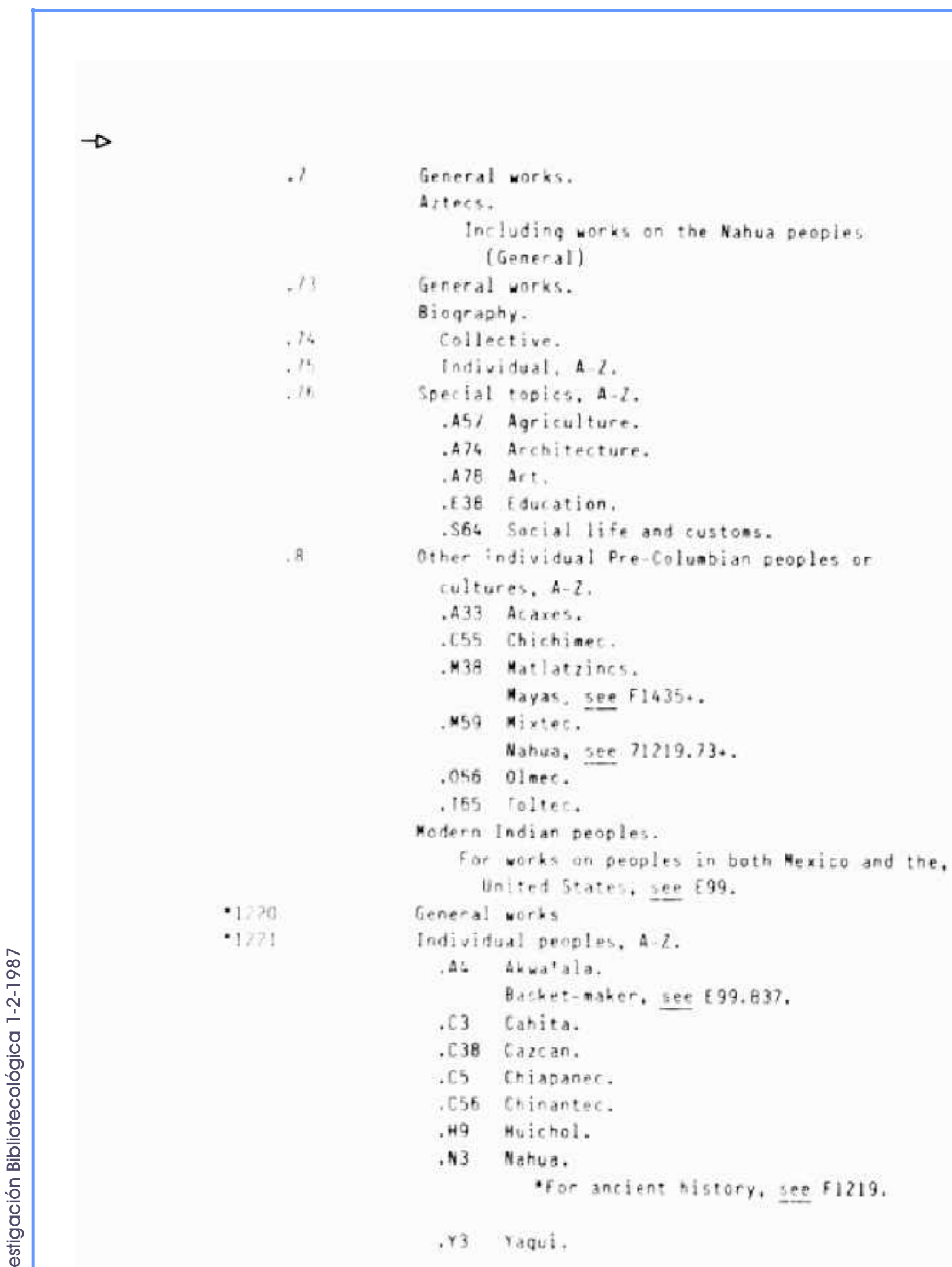


CUNORO 3

HISTORta MEXtCANa

F MEXICO

History.

Historiography.

Genersl rorks.

Bisgraphy of historians.

-A2 Collective.

-43-2 Individeal.

General special

Military and asval history.

Diplanatic history (Genrral). Foreign and general telacions.

The Spanish conquest: Gortes and his companions: Bernal ofar del Castille; Montezuna It; etc. 1535-1810.

Period of the viceroys: church and state: expulsion of the Jesuits: itc.

Blography: Luis de Carvajal: Jose de Iturrigaray y Aróstegui: Cuillén Lobardo; Amtonio de Mendoza, conde de Tandilla: Juan de Palafox, Mendoza; Melcher de talanantes Salvador y Baeza.

Wars of Independente, 1810-1821; Eopice of Iturbide; troubles with france, 1838-1839; etc. Biography: Lucas Alanfin: Ignacio jost Allende Cf. [ 401-415, War vith-United States; Loss of New kexico and California.

$1849-1958 / 1861$.

Revolutions: state and church: Canstitution of 1857; Raousset-Boulbon and Malker sonora Expeditions: $t: c$.

Buagraphy: Juan Alvarez; Mariano Arista; Ignacio Cowonfort; Easton Raoux, conte RaoussetBoulbon; etc,

European intervention. 1864-1867. Feench aray it Mexico: Enpire of Maximilias, etc,

Biography; Chorlotte, cansort of Maxililian: Mariano Escobeda: Benite Pablo Juárez: Maximilian, eaperor of Mexico; etc.

Gadsden Treaty. Sale of territory sauth of the Gila to the United States. See f 786 . 


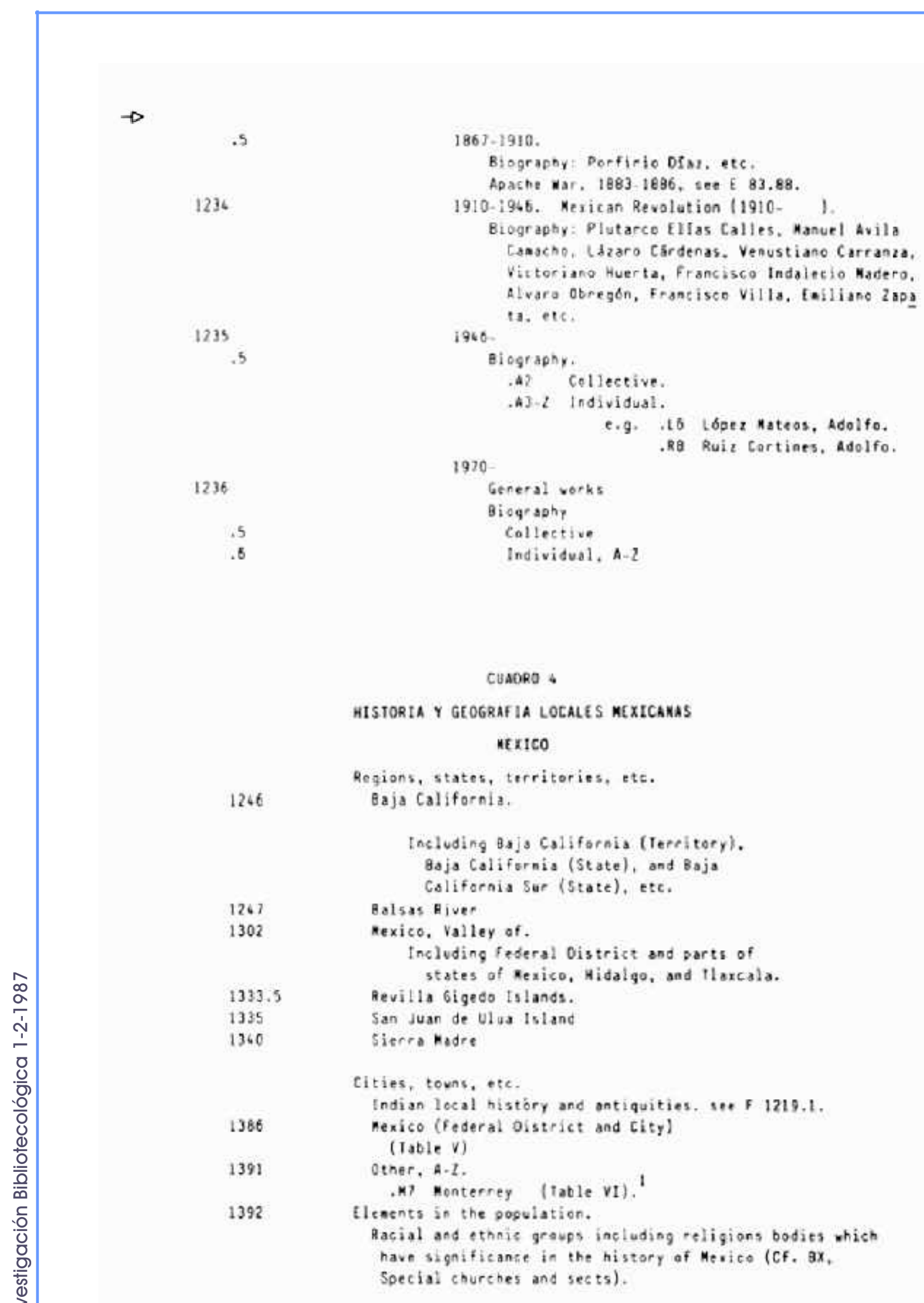


TABLES FOR CITIES WIIK SIMGLE NUMECA OR CJITER NIMMBER, IN UNITED STATES, BRIIISH AMERICA ANO IATIN AMERICA

To be used only where indicated in schedules.

$v^{1} \quad v t^{2}$

- A2 -x Periodicals. Societies. Coilectians.

. A3 $\quad \times 2$

Museums. Exhibitions, exhabits.

$.46 \quad \times 3$

. AS- $2 \quad . \times 6$

$.1 \times 43$

$.13 \quad . \times 45$

$.15 \quad . \times 47$

$.2 \quad \times 5$

$.23-\times 53$

$.25 \quad \times 55$

$\times 3 \times 5$ ?

$.4 \times 6$

$.5 \quad . \times 65$

$-6 \quad \times 7$

$.7 \quad \times \times 75$

$.8 \quad \times 8$

Guidebooks. Gazetteers. Directories.

General works. Description.

Monumental and picturesgur.

Minor works. Pamphlets, addresses, exsays. Antiquities.

Social life and tustoms. Intellectual jife. History.

Biography.

Historiegraphy. Study and teaching.

General witks.

Sections. Di-tricts getc. 3

Parks. Squares. Circles.

Streets. ${ }^{3}$

Buildings.

Elements in the population.

9.A2A-2 $\because \times 9 A 2-29$

General. Collective.

$.9 . A 3-2 \quad \times \times 9 R 3-2$

Individual. A 2 .

For lists of Cutters, see $\mathrm{E} 1 \mathrm{~B} 4$. United States: F1035, Canada;

F1342, Mexico; 51677, Guatenala:

etc.

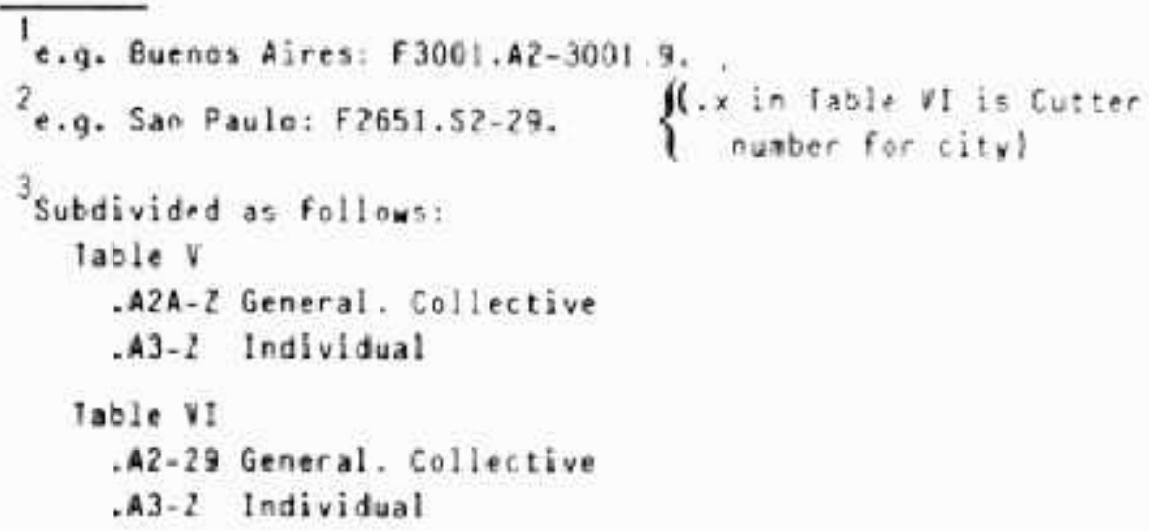


CUADRO 3

MODIF ICACION AL ESOUEAA PARA HISTORTA Y GEOGRAFIA LOCALES MEXICANAS

F

MEXICO

f

Hegians, atutes, territories, etc.

1376 Yucatan. Indian rulns (General).

Indian history and local sntiquitiss, ses f 1219.1. Local

Mayan antiquities, set $r$ 1435.1.

Cf. I 1449.87, Beftish Handuras boundary.

TABLES FOR COUNTRIES, ISLANDS, REGIONS

WITH SINGLE NUMBER DA CUTTER NUMBER

10 be used only there indicated in sehedules.

\begin{tabular}{|c|c|c|}
\hline$v_{11}{ }^{\circ}$ & $v H^{5}$ & Ix \\
\hline $.42 A-2$ &.$\times A ?$ & $x+2=$ \\
\hline . $A 3 A-2$ &.$\times A 3.39$ &,$x \mathrm{~A} 3-$ \\
\hline .452 & $\therefore A S 2$ & $\therefore$ A $5=$ \\
\hline .2 &.$\times 2$ & $1 \times 2$ \\
\hline .3 &.$\times 3$ & $\therefore 3$ \\
\hline+4 & $+x$ & $1 \times 6$ \\
\hline .42 &.$\times 42$ & $x+2$ \\
\hline .5 & $x^{5}$ & .55 \\
\hline .5 & .66 & $\times 6$ \\
\hline 52 & $+x \in ?$ & $\therefore 6 ?$ \\
\hline .53 &. .63 &.$\times 63$ \\
\hline
\end{tabular}

Poriodicals. Sacieties.

Sources and docunents. Collections.

General verks.

Description and travel. Guidebooks.

Gazetteers.

Antiquities, Indians.

Sacial life and custons. Civiliza-

tion.

Elenents in the population.

History

General works

Biography and menoits (Collective)

Poliftical and diplonatic history

General wnrks

Relations with individual countries, A- 2

Far list of countries, see PP. $605-606$

By Deriod

Early

$.65 \quad .065 \quad . \times 65$

General works

Bisgraphy and nemoirs

follective

Indiviousl. A-Z

.67 $-x 67 \quad-x+7$

Colonia:

General works

Bisgraphy and nemeirs

collective

Individual. A-t

Independent

General works

Bisgraphy sod anteirs

Collectirs

Individual, A-2

local, A-? 